DOI: $10.3901 / J M E .2021 .17 .163$

\title{
定模动辊机电系统动力学分析*
}

\author{
阳振峰 1,2 李 强 1,2 管延智 ${ }^{1,2}$ 陈晓宇 ${ }^{1,2}$ 朱越坤 ${ }^{1,2}$ 李傲然 1,2
}

(1. 北方工业大学机械与材料工程学院 北京 100144 ;

2. 北京市变截面辊弯成形工程技术研究中心 北京 100144)

\begin{abstract}
摘要: 动力学分析对定模动辊成形装备的设计具有重要意义。论文基于能量原理, 针对驱动电动机、传动机构、模具-轧辊系 统与成形高强钢板材组成的机电系统, 提出一种包含电磁场、机构驱动力场及高强钢板连续塑性变形过程动态能量场耦合机 电系统动力学分析新方法。通过推导系统各部分能量数学表达的理论研究, 获得定模动辊机电系统动力学方程。采用有限元 法获取定模动辊成形过程中的反力矩, 以此作为载荷并用 Runge-Kutta 法求解系统微分方程, 揭示了驱动电动机、传动机构、 模具-轧辊系统与先进高强钢板材变形组成的系统运动机理, 全方位展示装备动特性规律, 求得各轴载荷及电动机驱动力矩等 系统性能参数, 实现先进高强钢变截面定模动辊成形工况匹配设计与精确运动控制。设计的定模动辊成形机与分析结果进行 了可行性实验验证。
\end{abstract}

关键词: 定模动辊; 能量原理; 机电系统; 动力学分析

中图分类号: TG156

\section{Electromechanical Dynamics Analysis of Roll-Die Forming Machine}

\author{
YANG Zhenfeng $^{1,2}$ LI Qiang ${ }^{1,2}$ GUAN Yanzhi ${ }^{1,2}$ CHEN Xiaoyu $^{1,2}$ ZHU Yuekun ${ }^{1,2}$ LI Aoran ${ }^{1,2}$ \\ (1. School of Mechanical and Materials Engineering, North China University of Technology, Beijing 100144 \\ 2. Beijing Research Center of Flexible Roll Forming Engineering, Beijing 100144)
}

\begin{abstract}
Dynamic analysis is of great significance to the design of roll-die forming machine. Aiming at the electromechanical system composed of drive motor, drive mechanism, roll-die system and formed high strength steel plate, based on the energy principle, a new dynamic analysis method of electromechanical system including electromagnetic field, driving force field of mechanism and dynamic energy field in continuous plastic deformation process of high strength steel plate is proposed. Through theoretical research, the energy mathematical expression of each part of the system is deduced, and then the electromechanical dynamics equation of the roll-die forming machine is obtained. The counter torque in the process of roll-die forming is extracted by finite element method, and be used to solve the differential equations by Runge Kutta method. The motion mechanism of the system composed of driving motor, driving mechanism, roll-die system and advanced high strength steel sheet deformation is revealed. The system performance parameters such as the load of each axis and the driving torque of the motor are obtained. The servo motion speed and acceleration of each roll show the dynamic characteristics of the equipment in an all-round way, so as to realize the performance design and accurate control for advanced high strength steel variable cross-section roll-die forming machine. The feasibility of the developed roll-die forming machine is verified by the analysis results
\end{abstract}

Key words: roll-die forming machine; energy principle; electromechanical system; dynamical analysis

\section{0 前言}

先进高强钢屈服极限高, 延伸率较低。其变截
面薄板构件，在载荷一定条件下轻量化性能显著， 成为汽车、高速列车等工业追求的目标。先进高强 钢高强度、低延伸率特性决定了渐进成型是其可行 的成形方法。等截面辊弯成形是成熟的渐进成型技 术。但先进高强钢杆类构件变截面辊弯成形, 是工 程领域正在探索的新问题。德国科学家

* 国家自然科学基金资助项目(51775004)。20200915 收到初稿, 20201225 收到修改稿 
INGVARSON 教授于 2002 年提出变截面辊弯成形 的概念。2010 年, GROCHE ${ }^{[1]}$ 等针对变截面辊弯成 形提出了单道次模型分析法, 实现了变截面辊弯成 形过程快速验证。日本拓殖大学针对变截面辊弯成 形技术与实现方法开展相关研究 ${ }^{[2]}$, 并于 2010 年在 试验室完成单侧变截面辊弯成形样机。德国 Darmstadt 大学(Technische Universität Darmstadt)在 辊弯成形方面做了许多工作。德国 dataM 公司和瑞 典 Ortic 公司分别于 2010 年、 2012 年研制成功针对 某种变截面构件的辊弯成形样机。PARALIKAS ${ }^{[3]}$ 等针对先进高强钢 $U$ 形对称截面辊弯成形, 选择板 料速度、辊组间距、辊缝和轧辊直径为影响因素, 完成了等截面辊弯成形生产线系统优化。北方工业 大学从 2005 年开始进行变截面辊弯成形研究。2009 年研制成功 9 道次单侧变截面辊弯成形样机, 并实 现普碳钢板材单侧变截面辊弯成形样件制造。2013 年完成三维辊弯成形样机研制, 并实现高强钢 TRIP590 汽车 B 柱下部加强板变截面构件辊弯成 形。2013 年提出定模动辊成形新技术, 2014 年完成 定模动辊生产线样机研制, 2016 年 11 月完成变高度 定模动辊生产线研制, 并实现 DP980 先进高强钢变 截面辊弯成形样件制造。同时, 开展针对三维辊弯成 形样机的装备系统性能研究, 相关成果见文献[4-6]。

先进高强钢辊弯成形过程中成形力巨大, 经有 限元分析, 对于抗拉强度 $1200 \mathrm{MPa}, 1.5 \mathrm{~mm}$ 厚的 高强钢板, 变截面辊弯成形的动态横向力载荷最高 可达 $14 \mathrm{kN}$ 。大的成形力导致辊弯成形设备的结构 增大, 轧辊回转空间增大, 无法实现构件的精细特 征辊弯成形。同时伺服运动的驱动电动机载荷增大, 变截面辊弯成形运动迟滞效应显著, 不同道次的伺 服电动机负载间形成较大差异, 导致各道次轧辊的 伺服运动难以实现相应的理论进给量和各道次间需 要精确的协调运动。由此可见, 先进高强钢变截面 辊弯成形的实现, 需要解决涉及大塑性成形力和大 规模复杂联动与协调运动为一体的制造装备问题 [7]。本文作者团队融合型模和多轴联动, 空间协同 运动的自随形精确成形技术, 从提高变截面辊弯成 形精度角度提出定模动辊变截面辊弯成形新方法, 有效地解决了变截面辊弯成形领域的难题。该方法 获得 PCT 美国发明专利 ${ }^{[8]}$ 。

实现定模动辊变截面辊弯成形工程应用, 就需 要针对不同先进高强钢变截面构件, 设计相应的定 模动辊变截面辊弯成形生产线。因此定模动辊成形 装备性能研究, 特别是系统动力学研究成为装备设 计的首要任务。综合考虑不同种类先进高强钢板材
变截面构件定模动辊成形过程中不同成形速度下动 态载荷, 系统控制条件下定模及所配置的动辊组机 构动态性能, 获得系统综合性能最优, 决定工程应 用开发的快捷性与成功率。定模动辊成形装备的设 计制造, 变形抗力是必要参数。利用各道次各轧辊 动态负载设置控制参数, 实现设定的系统运动。对 于这种瞬态变形, 变形抗力与变形程度和变形速率 有关 ${ }^{[9]}$, 并受应变速率影响。本文以变截面定模动 辊成形装备设计为目标, 开展装备系统动力学理论 研究, 揭示先进高强钢变截面定模动辊装备驱动电 动机、传动机构、模具-轧辊系统与变形板材组成的 系统运动机理, 获得各轴载荷及电动机驱动力矩等 系统设计参数及包含各轧辊伺服运动速度、加速度 的动特性规律, 实现先进高强钢变截面辊弯工况匹 配设计与精确成形控制。为变截面辊弯成形生产线 的研制提供系统设计理论。

\section{1 定模动辊成形机组成}

变高度定模动辊成形机主要包括 $X$ 向运动大机 架、 5 个 $Z$ 向运动小机架、模具及实现模具开合的 液压气动元件等组成。 $X$ 向驱动电动机通过齿轮齿 条驱动 $X$ 向运动机架, 带动 5 个道次整体向前运动, 轧辊驱动电动机驱动轧辊旋转。 $Z$ 向驱动电动机负 责调整轧辊垂向位置, 如图 1 所示为变高度定模动 辊成形机三维图。

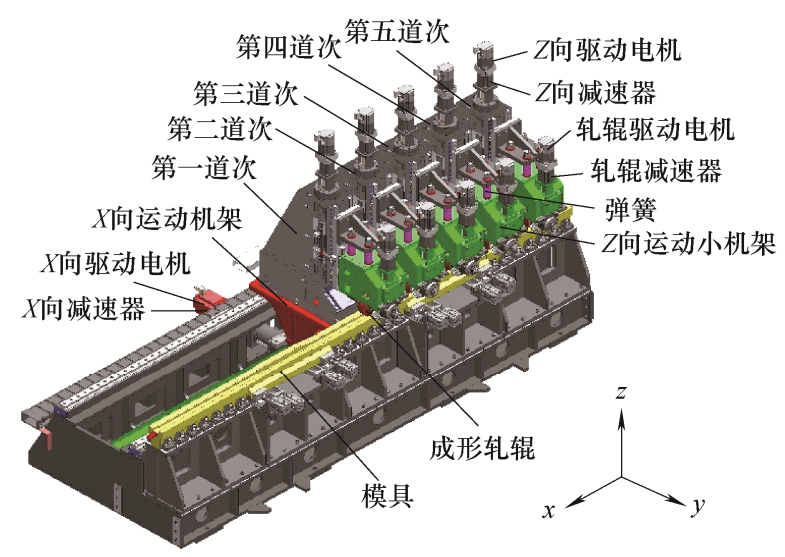

图 1 变高度定模动辊成形机三维图

针对成形过程中辊组沿模具运动建立坐标系, 可将变高度定模动辊成形机的运动简化为如图 2 所 示。轧辊可在 $x 、 z$ 向平动并绕 $y$ 向转动, 实现沿模 具的变截面渐进成形。

成形板料静止于模具, 轧辊组以图 2 所示速度 方向依次沿模具滚动, 轧辊的形状决定了每个道次 不同的折弯角度。根据定模动辊成形工艺设定 5 个 
道次成形角度为 $30^{\circ}, 50^{\circ}, 70^{\circ}, 84^{\circ}, 84^{\circ}$ 。

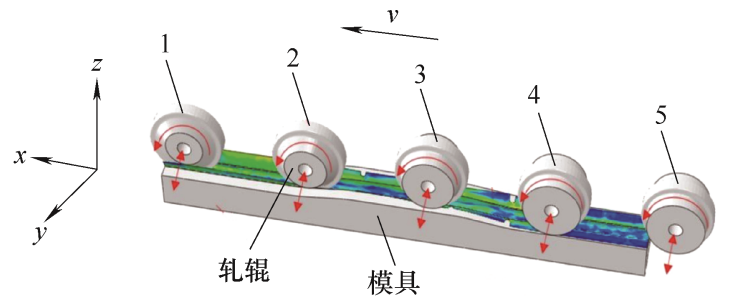

图 2 变高度定模动辊成形机的运动简图

\section{2 能量原理}

机电耦联系统由机械系统、电系统和联系两者 的耦合电磁场组成。电系统为伺服电动机的驱动电 压和电流, 耦合电磁场由伺服电动机特性决定。由 能量守恒原理 ${ }^{[10]}$, 可得出机电耦联系统能量关系的 微分形式为

$$
\mathrm{d} W_{\mathrm{E}}=\mathrm{d} W_{\mathrm{F}}+\mathrm{d} W_{\text {out }}
$$

式中, $\mathrm{d} W_{\mathrm{E}} 、 \mathrm{~d} W_{\mathrm{F}} 、 \mathrm{~d} W_{\text {out }}$ 分别为时间 $\mathrm{d} t$ 内输入耦 合场的净电能、耦合场吸收的总能量以及机电耦合 系统输出的总能量。

$$
\mathrm{d} W_{\mathrm{E}}=\sum_{j} \boldsymbol{U}_{j}^{\mathrm{T}} \boldsymbol{I}_{j} \mathrm{~d} t
$$

式中 $\boldsymbol{U}_{j} 、 \boldsymbol{I}_{j}$ 分别为第 $j$ 个电动机的定子三相电 压、电流。

$$
\mathrm{d} W_{\mathrm{F}}=\mathrm{d} W_{\mathrm{m}}+\mathrm{d} W_{\mathrm{e}}
$$

式中, $W_{\mathrm{m}}$ 为电磁场内的磁能, $W_{\mathrm{e}}$ 为电能。

驱动电动机耦合场的输出总能量等于系统中所 有电磁力做功的总和。对于第 $k$ 点形成微位移为 $\mathrm{d} x$ 的电磁力 $f_{\mathrm{e}}$, 当任意其他点 $j \neq k$ 时, $d x_{j}=0$, 因 此可得

$$
\mathrm{d} W_{\text {out }}=\sum_{k}\left(f_{\mathrm{e}}\right)_{k} \cdot \mathrm{d} x_{k}=\sum_{k}\left(f_{\mathrm{e}}\right)_{k} \dot{x}_{k} \mathrm{~d} t=\mathrm{d} T+\mathrm{d} W_{\mathrm{s}}
$$

式中, $T$ 为系统中动能和势能, $W_{\mathrm{s}}$ 为系统中的机电 损耗。

将式(2) (4)代入式(1)可得电磁力所做功的表 达式为

$$
\sum_{k}\left(f_{\mathrm{e}}\right)_{k} \dot{x}_{k} \mathrm{~d} t=\sum_{j} \boldsymbol{U}_{j}^{\mathrm{T}} \boldsymbol{I}_{j} \mathrm{~d} t-\mathrm{d} W_{\mathrm{m}}-\mathrm{d} W_{\mathrm{e}}
$$

则基于能量原理的机电耦联系统动力学分析的 数学表达为

$$
\sum_{j} \boldsymbol{U}_{j}^{\mathrm{T}} \boldsymbol{I}_{j} \mathrm{~d} t=\mathrm{d} W_{\mathrm{m}}+\mathrm{d} W_{\mathrm{e}}+\mathrm{d} W_{\mathrm{s}}+\mathrm{d} T
$$

系统中动能用于实现定模动辊的塑性成形。

\section{3 定模动辊系统动力学建模与计算}

\section{1 确定广义坐标}

定模动辊机电耦联系统包括 11 个伺服电动机和 5 个辊组机构。选取广义坐标如下, $e_{a j} 、 e_{b j} 、 e_{c j}$ 为第 $j(j=1,2, \cdots, 11)$ 个电动机的定子三相绕组的电量; $\boldsymbol{I}_{j}$ 、 $\boldsymbol{U}_{j} 、 \boldsymbol{R}_{s j}$ 分别为第 $j$ 个伺服电动机定子三相电流、电 压、电阻的矩阵, $\varphi_{j} 、 \dot{\varphi}_{j}$ 为第 $j$ 个电动机的主轴转角 和转角速度, $B_{j}$ 为伺服电动机的阻尼系数, $T_{f_{k}}$ 为第 $k(k=1,2,3,4,5)$ 个轧辊所受的成形力矩。

\section{2 计算系统输出的各部分能量}

(1) 大机架 $x$ 向运动增加的动能 $T_{x}$

记 $X$ 向驱动电动机的转动惯量和主轴转角分别 为 $J_{x 1} 、 \varphi_{x}, X$ 向减速器的转动惯量及减速比分别 为 $J_{x 2} 、 i_{x}, X$ 向联轴器的转动惯量为 $J_{x 3}, X$ 向驱 动齿轮的转动惯量及分度圆半径分别为 $J_{x 4} 、 R_{x}$, 包含五道次的 $X$ 向运动大机架质量为 $m_{x}$, 则大机架 $x$ 向运动增加的动能 $T_{x}$ 可表示为

$$
\begin{aligned}
T_{x}= & \frac{1}{2}\left(J_{x 1}+J_{x 2}\right) \dot{\varphi}_{x}^{2}+\frac{1}{2 i_{x}^{2}}\left(J_{x 3}+J_{x 4}\right) \dot{\varphi}_{x}^{2}+\frac{1}{2} m_{x}\left(\frac{R_{x} \dot{\varphi}_{x}}{i_{x}}\right)^{2}= \\
& \frac{1}{2}\left[J_{x 1}+J_{x 2}+\frac{1}{i_{x}^{2}}\left(J_{x 3}+J_{x 4}+m_{x} R_{x}^{2}\right)\right] \dot{\varphi}_{x}^{2}
\end{aligned}
$$

(2) 五个 $Z$ 向运动小机架增加的势能与动能之 和 $T_{z}$ 。

记 $Z$ 向驱动电动机的转动惯量及主轴转角分别 为 $J_{z_{1}} 、 \varphi_{z_{k}}(k=1,2,3,4,5), Z$ 向减速器转动惯量及 减速比分别为 $J_{z_{2}} 、 i_{z}, Z$ 向联轴器的转动惯量为 $J_{z_{3}}, Z$ 向运动小机架滚珠丝杜的转动惯量及导程分 别为 $J_{z_{4}} 、 S, Z$ 向运动小机架与自适应机架总质量 为 $m_{z}$, 则五个 $Z$ 向运动小机架在 $Z$ 向电动机驱动下 增加的沿 $z$ 向移动的势能与动能之和 $T_{z}$ 可表示为

$$
\begin{aligned}
T_{z}= & \frac{1}{2}\left(J_{z_{1}}+J_{z_{2}}\right) \sum_{k=1}^{5} \dot{q}_{z_{k}}^{2}+\frac{1}{2 i_{z}^{2}}\left(J_{z_{3}}+J_{z_{4}}\right) \dot{\varphi}_{z_{k}}^{2}+ \\
& \frac{1}{2} m_{z}\left(\frac{S \sum_{k=1}^{5} \dot{\varphi}_{z_{k}}}{2 \pi i_{x}}\right)^{2}+m_{z} g \frac{S}{2 \pi i_{z}} \dot{\varphi}_{z_{k}}= \\
& \frac{1}{2}\left[J_{z_{1}}+J_{z_{2}}+\frac{1}{i_{z}^{2}}\left(J_{z_{3}}+J_{z_{4}}+m_{x} \frac{S^{2}}{4 \pi^{2}}\right)\right] \sum_{k=1}^{5} \dot{\varphi}_{z_{k}}^{2}+m_{z} g \frac{S}{2 \pi i_{z}} \sum_{k=1}^{5} \dot{\varphi}_{z_{k}}
\end{aligned}
$$

(3) 五道次车辊在电动机驱动下增加的转动动能 $T_{r}$ 。 记轧辊驱动电动机的转动惯量及主轴转角分别 为 $J_{r_{1}} 、 \varphi_{r_{k}}(k=1,2,3,4,5)$, 轧辊减速器的转动惯量 及减速比分别为 $J_{r_{2}} 、 i_{r_{1}}$, 轧辊驱动联轴器的转动惯 
量为 $J_{r_{3}}$, 轧辊驱动齿轮、从动齿轮的转动惯量分别 为 $J_{r_{4}} 、 J_{r_{5}}$, 雉齿轮副的传动比为 $i_{r_{2}}$, 轧辊的转动 惯量为 $J_{r_{6}}$, 则五道次轧辊在电动机驱动下增加的转 动动能 $T_{r}$ 可表示为

$$
\begin{aligned}
T_{r}= & \frac{1}{2}\left(J_{r_{1}}+J_{r_{2}}\right) \sum_{k=1}^{5} \dot{\varphi}_{r_{k}}^{2}+\frac{1}{2 i_{r_{1}}^{2}}\left(J_{r_{3}}+J_{r_{4}}\right) \sum_{k=1}^{5} \dot{\varphi}_{r_{k}}^{2} \\
& +\frac{1}{2 i_{r_{1}}^{2} i_{r_{2}}^{2}}\left(J_{r_{5}}+J_{r_{6}}\right) \sum_{k=1}^{5} \dot{\varphi}_{r_{k}}^{2} \\
=\frac{1}{2}\left[J_{r_{1}}\right. & \left.+J_{r_{2}}+\frac{1}{i_{r_{1}}^{2}}\left(J_{r_{3}}+J_{r_{4}}\right)+\frac{1}{i_{r_{1}}^{2} i_{r_{2}}^{2}}\left(J_{r_{5}}+J_{r_{6}}\right)\right] \sum_{k=1}^{5} \dot{\varphi}_{r_{k}}^{2}
\end{aligned}
$$

(4) 轧辊克服成形力做的功 $W_{T_{f}}$ 。

将获得的五道次轧辊反作用力矩 $T_{f_{k}}$ 作为成形 过程中受到的滚阻力矩, 则轧辊克服成形力做的功 可表示为

$$
W_{T_{f}}=\sum_{k=1}^{5} \frac{\varphi_{r_{k}}}{i_{r_{1}} i_{r_{2}}} T_{f_{k}}
$$

(5) 机械系统的耗散能 $W_{f}$ 。

记 $Z$ 向运动小机架的滚动直线导轨副及滚珠丝杜 副的阻尼系数分别为 $c_{1} 、 c_{A}$ ，第 $k$ 道次小机架沿 $z$ 向 坐标运动的位移为 $z_{k}$, 机械系统耗散能 $W_{f}$ 可表示为

$$
W_{f}=\frac{1}{2} c_{1} \sum_{k=1}^{5}\left(\dot{z}_{k}\right)^{2}+\frac{1}{2 i_{z}^{2}} c_{A} \sum_{k=1}^{5}\left(\dot{\varphi}_{z_{k}}\right)^{2}
$$

记 $X$ 向驱动齿轮的分度圆半径为 $R_{x}$, 则综上分 析可得轧辊成形过程中机械系统能量为

$$
\begin{aligned}
T= & T_{x}+T_{z}+T_{r}+W_{T_{f}}+W_{f}= \\
& \frac{1}{2}\left[J_{x_{1}}+J_{x_{2}}+\frac{1}{i_{x}^{2}}\left(J_{x_{3}}+J_{x_{4}}+m_{x} R_{x}^{2}\right)\right] \dot{\varphi}_{x}^{2}+ \\
& \frac{1}{2}\left[J_{z_{1}}+J_{z_{2}}+\frac{1}{i_{z}^{2}}\left(J_{z_{3}}+J_{z_{4}}+m_{z} \frac{S^{2}}{4 \pi^{2}}\right)\right] \sum_{k=1}^{5} \dot{\varphi}_{z_{k}}^{2}+ \\
& m_{z} g \frac{S}{2 \pi i_{z}} \sum_{k=1}^{5} \varphi_{z_{k}}+\frac{1}{2}\left[J_{r_{1}}+J_{r_{2}}+\frac{1}{i_{r_{1}}^{2}}\left(J_{r_{3}}+J_{r_{4}}\right)+\right. \\
& \left.\frac{1}{i_{r_{1}}^{2} i_{r_{2}}^{2}}\left(J_{r_{5}}+J_{r_{6}}\right)\right] \sum_{k=1}^{5} \dot{\varphi}_{r_{k}}^{2}+\sum_{k=1}^{5} \frac{\varphi_{r_{k}}}{i_{r_{1}} i_{r_{2}}} T_{f_{k}}+ \\
& \frac{1}{2} c_{1} \sum_{k=1}^{5}\left(\dot{z}_{k}\right)^{2}+\frac{1}{2 i_{z}^{2}} c_{A} \sum_{k=1}^{5}\left(\dot{\varphi}_{r_{k}}^{2}\right)^{2}
\end{aligned}
$$

记轧辊沿系统坐标系 $x$ 轴移动的位移为 $x$, 第 $k$ 道次变高度轧辊轴线与 $x$ 轴夹角为 $\beta_{k}(t)$, 轧辊半径 为 $R_{r}$, 则伺服电动机主轴转角量可用系统坐标系三 个方向的运动位移量表示。

$$
\left\{\begin{array}{l}
\varphi_{z_{k}}=\frac{2 \pi i_{z} \tan \beta_{k}}{S} x \\
\varphi_{x}=\frac{i_{x}}{R_{x}} x \\
\varphi_{r_{k}}=\frac{i_{r_{1}} i_{r_{2}}}{R_{r} \cos \beta} x
\end{array}\right.
$$

将(13)代入式(12), 机械系统增加的能量简化为 $T=\frac{1}{2}\left(J_{x}+J_{z}+J_{r}\right) \dot{x}^{2}+\left(m_{z} g \sum_{k=1}^{5} \tan \beta_{k}+\sum_{K=1}^{5} \frac{T_{f_{k}}}{R_{r} \cdot \cos \beta_{k}}\right) x$

式中

$$
\left\{\begin{aligned}
J_{x}= & \frac{i_{x}^{2}}{R_{x}^{2}}\left(J_{x_{1}}+J_{x_{2}}\right)+\frac{1}{R_{x}^{2}}\left(J_{x_{3}}+J_{x_{4}}\right)+m_{x} \\
J_{z}= & {\left[\left(\frac{2 \pi i_{z}}{S}\right)^{2}\left(J_{z_{1}}+J_{z_{2}}\right)+\left(\frac{2 \pi}{S}\right)^{2}\left(J_{z_{3}}+J_{z_{4}}\right)+m_{z}\right] \cdot \sum_{k=1}^{5}\left(\tan ^{2} \beta\right) } \\
J_{r}= & {\left[\left(\frac{i_{r_{1}} i_{r_{2}}}{R_{r}}\right)^{2}\left(J_{r_{1}}+J_{r_{2}}\right)+\left(\frac{i_{r_{2}}}{R_{r}}\right)^{2}\left(J_{r_{3}}+J_{r_{4}}\right)+\left(\frac{1}{R_{r}}\right)^{2}\left(J_{r_{5}}+J_{r_{6}}\right)\right] } \\
& \cdot \sum_{k=1}^{5}\left(\frac{1}{\cos ^{2} \beta_{k}}\right)
\end{aligned}\right.
$$

\section{3 电磁系统损耗的能量}

考虑系统伺服电动机电磁力做功过程的电磁损 耗 ${ }^{[11]}$, 记 $\boldsymbol{I}_{j} 、 \boldsymbol{R}_{\mathrm{s} j}$ 分别第 $j(j=1,2, \cdots, 11)$ 个伺服电动 机的定子三相电流矩阵和电阻矩阵, $B_{x}$ 为 $X$ 向驱动电 动机的转子黏滞阻尼系数。记型号相同的五道次 $Z$ 向 驱动电动机的转子粘滞阻尼系数为 $B_{z}$, 型号相同的五 道次轧辊驱动电动机的转子黏滞阻尼系数为 $B_{r}$, 电动 机电阻能量损耗以矩阵相乘形式表达, 则系统电磁损 耗能量为

$$
W_{\mathrm{s}}=\frac{1}{2} \sum_{j=1}^{11} \boldsymbol{I}_{j}^{\mathrm{T}} \boldsymbol{R}_{\mathrm{s} j} \boldsymbol{I}_{j}+\frac{1}{2}\left(B_{x} \dot{\varphi}_{x_{k}}^{2}+B_{z} \sum_{k=1}^{5} \dot{\varphi}_{z_{k}}^{2}+B_{r} \sum_{k=1}^{5} \dot{\varphi}_{r_{k}}^{2}\right)
$$

为便于运算, 将伺服电动机主轴转角量用系统 坐标系三个方向的运动位移量表示, 并通过 $K_{3 s / 2 r}$ 矩 阵将上式从自然坐标系 $a b c$ 变换为同步旋转坐标系 $d-q$, 则电磁损耗能量表达式为

$$
\begin{gathered}
W_{\mathrm{s}}=\frac{3}{4} \sum_{j=1}^{11}\left[r_{\mathrm{s}}\left(i_{d}^{2}+i_{q}^{2}\right)\right]_{j}+\frac{1}{2}(B+C) \dot{x}^{2} \\
\left\{\begin{array}{l}
B=B_{x} \frac{i_{x}^{2}}{R_{x}^{2}}+B_{z}\left(\frac{2 \pi i_{z} \sum_{k=1}^{5} \tan \beta_{k}}{S}\right)^{2}+B_{r} \sum_{k=1}^{5}\left(\frac{i_{r_{1}} i_{r_{2}}}{R_{r} \cos \beta_{k}}\right)^{2} \\
C=\left[c_{1}+c_{A}\left(\frac{2 \pi}{S}\right)^{2}\right] \sum_{k=1}^{5} \tan \beta_{k}
\end{array}\right.
\end{gathered}
$$

\section{4 机电系统动力学微分方程组}

定模动辊系统耦合磁场增加的磁能为

$$
W_{\mathrm{m}}=\frac{1}{2} \sum_{j=1}^{11} \boldsymbol{I}_{j}^{\mathrm{T}} \boldsymbol{L}_{j} \boldsymbol{I}_{j}+\sum_{j=1}^{11} \boldsymbol{I}_{j}^{\mathrm{T}} \boldsymbol{\psi}_{f_{j}}
$$

系统耦合场增加的电能为

$$
W_{\mathrm{e}}=0
$$

为方便运算, 将电能与磁能从自然坐标系 $a b c$ 
变换为同步旋转坐标系 $d-q$

$$
\boldsymbol{K}_{3 s / 2 r}=\frac{2}{3}\left[\begin{array}{ccc}
\cos \left(\theta_{r}\right) & -\sin \left(\theta_{r}\right) & 1 \\
\cos \left(\theta_{r}-2 \pi / 3\right) & -\sin \left(\theta_{r}-2 \pi / 3\right) & 1 \\
\cos \left(\theta_{r}+2 \pi / 3\right) & -\sin \left(\theta_{r}+2 \pi / 3\right) & 1
\end{array}\right]
$$

变换后系统输入的电能与磁场增加的磁能可表 示为

$$
\begin{gathered}
\sum_{j=1}^{11} \boldsymbol{U}_{j}^{\mathrm{T}} \boldsymbol{I}_{j} \mathrm{~d} t=\frac{3}{2} \sum_{j=1}^{11}\left(u_{d_{j}} i_{d_{j}}+u_{q_{j}} i_{q_{j}}\right) \mathrm{d} t \\
W_{\mathrm{m}}=\frac{3}{2} \sum_{j=1}^{11}\left(L_{d_{j}} i_{d_{j}} \dot{i}_{d_{j}}+L_{q_{j}} i_{q_{j}} \dot{i}_{q_{j}}+\psi_{f_{i}} \dot{i}_{d_{j}}\right)
\end{gathered}
$$

式(14)、(17)、(23)对时间 $t$ 求导后与式(22)一并 代入式(6), 则基于能量守恒原理的机电系统动力学 微分方程为

$$
\begin{aligned}
& \frac{3}{2} \sum_{j=1}^{11}\left(u_{d_{\mathrm{j}}} i_{d_{j}}+u_{q_{j}} i_{q_{j}}\right)-\frac{3}{2} \sum_{j=1}^{11}\left(L_{d_{j}} i_{d_{j}} \dot{i}_{d_{j}}+L_{q_{j}} i_{q_{j}} \dot{i}_{q_{j}}+\psi_{f_{j}} \dot{i}_{d_{j}}\right) \\
& =\left(J_{x}+J_{z}+J_{r}+B+c\right) \dot{x} \ddot{x}+\left(m_{z} g \sum_{k=1}^{5} \tan \beta_{k}+\frac{1}{R_{r}} \sum_{k=1}^{5} \frac{T_{f_{i}}}{\cos \beta_{k}}\right) \dot{x} \\
& \quad+\frac{3}{2} r_{s} \sum_{j=1}^{11}\left(i_{d_{j}} \dot{i}_{d_{j}}+i_{q_{j}} \dot{i}_{q_{j}}\right)
\end{aligned}
$$

上式微分方程含 23 个未知数, 第 $j$ 个伺服电动 机的能量回路方程可表示为

$$
\left\{\begin{array}{l}
L_{d_{j}} \dot{i}_{d_{j}}-p_{j} \dot{\varphi}_{j} L_{q_{j}} i_{q_{j}}+i_{d_{j}} r_{s_{j}}=u_{d_{j}} \\
L_{q_{j}} \dot{i}_{q_{j}}-p_{j} \dot{\varphi}_{j} L_{d_{j}} i_{d_{j}}+p_{j} \dot{\varphi}_{j} \psi_{f_{j}}+i_{q_{j}} r_{q_{j}}=u_{q_{j}}
\end{array}\right.
$$

式(25)、(24)联立可得包含 11 个驱动电动机的 定模动辊机电系统动力学微分方程

$$
\left\{\begin{array}{l}
\frac{3}{2} \sum_{j=1}^{11}\left(u_{d_{j}} i_{d_{j}}+u_{q_{j}} i_{q_{j}}-L_{d_{j}} i_{d_{j}} i_{d_{j}}-L_{q_{j}} i_{q_{j}} i_{q_{j}}-\psi_{f_{j}} \dot{i}_{d_{j}}\right)- \\
\frac{3}{2} r_{s} \sum_{j=1}^{11}\left(i_{d_{j}} \dot{i}_{d_{j}}+i_{q_{j}} \dot{i}_{q_{j}}\right) \\
=\left(J_{x}+J_{z}+J_{r}+B+c\right) \dot{x} \ddot{x}+\left[m_{z} g \sum_{k=1}^{5}\left(\tan \beta_{k}\right)+\right. \\
\quad(j=1,2, \ldots, 11) \\
\left.\quad \frac{1}{R_{r}} \sum_{k=1}^{5} \frac{T_{f_{k}}}{\cos \beta_{k}}\right] \dot{x} \\
L_{d_{j}} i_{d_{j}}-p_{j} \dot{\varphi}_{j} L_{q_{j}} i_{q_{j}}+i_{d_{j}} r_{s_{j}}=u_{d_{j}} \\
L_{q_{j}} i_{q_{j}}+p_{j} \dot{\varphi}_{j} L_{d_{j}} i_{d_{j}}+p_{j} \dot{\varphi}_{j} \psi_{f_{j}}+i_{q_{j}} r_{s_{j}}=u_{q_{j}}
\end{array}\right.
$$

\section{4 机电系统动力学分析}

\section{1 系统动力学方程与参数确定}

系统动力学方程具体表达式为(27)。依据定模 动辊成形机设计参数, 所需的方程系数如表 1、2、 3 所示。

$$
\begin{aligned}
& \left(\frac{3}{2} \sum_{j=1}^{11}\left(u_{d_{j}} i_{d_{j}}+u_{q_{j}} i_{q_{j}}-L_{d_{j}} i_{d_{j}} i_{d_{j}}-L_{q_{j}} i_{q_{j}} i_{q_{j}}-\psi_{f_{j}} \dot{i}_{d_{j}}\right)-\frac{3}{2} r_{s} \sum_{j=1}^{11}\left(i_{d_{j}} \dot{i}_{d_{j}}+i_{q_{j}} \dot{i}_{q_{j}}\right)=\right. \\
& \left(J_{x}+J_{z}+J_{r}+B+c\right) \ddot{x} \ddot{x}+\left[m_{z} g \sum_{k=1}^{5}\left(\tan \beta_{k}\right)+\frac{1}{R_{r}} \sum_{k=1}^{5}\left(\frac{T_{f_{k}}}{\cos \beta_{k}}\right)\right] \dot{x} \\
& L_{d_{1}} \dot{i}_{d_{1}}-p_{1} L_{q_{1}} \frac{i_{x}}{R_{x}} \dot{x} i_{q_{1}}+r_{s_{1}} i_{d_{1}}=u_{d_{1}} \\
& L_{q_{1}} i_{q_{1}}+p_{1} L_{d_{1}} \frac{i_{x}}{R_{x}} \dot{x} i_{d_{1}}+\psi_{f_{1}} p_{1} \frac{i_{x}}{R_{x}} \dot{x}+r_{s_{1}} i_{q_{1}}=u_{q_{1}} \\
& L_{d_{2}} i_{d_{2}}-p_{2} L_{q_{2}} \frac{2 \pi i_{z} \cdot \tan \beta_{1}}{S} \dot{x} i_{q_{2}}+r_{s_{2}} i_{d_{2}}=u_{d_{2}} \\
& L_{q_{2}} i_{q_{2}}+p_{2} L_{d_{2}} \frac{2 \pi i_{z} \cdot \tan \beta_{1}}{S} \dot{x} i_{d_{2}}+\psi_{f_{2}} p_{2} \frac{2 \pi i_{z} \cdot \tan \beta_{1}}{S} \dot{x}+r_{s_{2}} i_{q_{2}}=u_{q_{2}} \\
& L_{d_{7}} \dot{i}_{d_{7}}-p_{7} L_{q_{7}} \frac{i_{r 1} \cdot i_{r 2}}{R_{r} \cdot \cos \beta_{1}} \dot{x} i_{q_{7}}+r_{s_{7}} i_{d_{7}}=u_{d_{7}} \\
& L_{q_{7}} i_{q_{7}}+p_{7} L_{d_{7}} \frac{i_{r_{1}} \cdot i_{r_{2}}}{R_{r} \cdot \cos \beta_{1}} \dot{x} i_{d_{7}}+\psi_{f_{7}} p_{7} \frac{i_{r_{1}} \cdot i_{r_{2}}}{R_{r} \cdot \cos \beta_{1}} \dot{x}+r_{s_{7}} i_{q_{7}}=u_{q_{7}} \\
& L_{d_{3}} i_{d_{3}}-p_{3} L_{q_{3}} \frac{2 \pi i_{z} \cdot \tan \beta_{2}}{S} \dot{x} i_{q_{3}}+r_{s_{3}} i_{d_{3}}=u_{d_{3}} \\
& L_{q_{3}} i_{q_{3}}+p_{3} L_{d_{3}} \frac{2 \pi i_{z} \cdot \tan \beta_{2}}{S} \dot{x} i_{d_{3}}+\psi_{f_{3}} p \frac{2 \pi i_{z} \cdot \tan \beta_{2}}{S} \dot{x}+r_{s_{3}} i_{q_{3}}=u_{q_{3}} \\
& L_{d_{8}} i_{d_{8}}-p_{8} L_{q_{8}} \frac{i_{r_{1}} \cdot i_{r_{2}}}{R_{r} \cdot \cos \beta_{2}} \dot{x} i_{q_{8}}+r_{s_{8}} i_{d_{8}}=u_{d_{8}} \\
& L_{q_{8}} \dot{i}_{q_{8}}+p_{8} L_{d_{8}} \frac{i_{r_{1}} \cdot i_{r_{2}}}{R_{r} \cdot \cos \beta_{2}} \dot{x} i_{d_{8}}+\psi_{f_{8}} p_{8} \frac{i_{r_{1}} \cdot i_{r_{2}}}{R_{r} \cdot \cos \beta_{2}} \dot{x}+r_{s_{8}} i_{q_{8}}=u_{q_{8}} \\
& L_{d_{4}} i_{d_{4}}-p_{4} L_{q_{4}} \frac{2 \pi i_{z} \cdot \tan \beta_{3}}{S} \dot{x} i_{q_{4}}+r_{s_{4}} i_{d_{4}}=u_{d_{4}} \\
& L_{q_{4}} i_{q_{4}}+p_{4} L_{d_{4}} \frac{2 \pi i_{z} \cdot \tan \beta_{3}}{S} \dot{x} i_{d_{4}}+\psi_{f_{4}} p_{4} \frac{2 \pi i_{z} \cdot \tan \beta_{3}}{S} \dot{x}+r_{s_{4}} i_{q_{4}}=u_{q_{4}} \\
& L_{d_{9}} i_{d_{9}}-p_{9} L_{q_{9}} \frac{i_{r_{1}} \cdot i_{r_{2}}}{R_{r} \cdot \cos \beta_{3}} \dot{x} i_{q_{9}}+r_{s_{9}} i_{d_{9}}=u_{d_{9}} \\
& L_{q_{9}} \dot{i}_{q_{9}}+p_{9} L_{d_{9}} \frac{i_{r_{1}} \cdot i_{r_{2}}}{R_{r} \cdot \cos \beta_{3}} \dot{x} i_{d_{9}}+\psi_{f_{9}} p_{9} \frac{i_{r_{1}} \cdot i_{r_{2}}}{R_{r} \cdot \cos \beta_{3}} \dot{x}+r_{s_{9}} i_{q_{9}}=u_{q_{9}} \\
& L_{d_{5}} i_{d_{5}}-p_{5} L_{q_{5}} \frac{2 \pi i_{z} \cdot \tan \beta_{4}}{S} \dot{x} i_{q_{5}}+r_{s_{5}} i_{d_{5}}=u_{d_{5}} \\
& L_{q_{5}} i_{q_{5}}+p_{5} L_{d_{5}} \frac{2 \pi i_{z} \cdot \tan \beta_{4}}{S} \dot{x} i_{d_{5}}+\psi_{f_{5}} p_{5} \frac{2 \pi i_{z} \cdot \tan \beta_{4}}{S} \dot{x}+r_{s_{5}} i_{q_{5}}=u_{q_{5}} \\
& L_{d_{10}} \dot{i}_{d_{10}}-p_{10} L_{q_{10}} \frac{i_{r_{1}} \cdot i_{r_{2}}}{R_{r} \cdot \cos \beta_{4}} \dot{x} i_{q_{10}}+r_{s_{10}} i_{d_{10}}=u_{d_{10}} \\
& L_{q_{10}} i_{q_{10}}+p_{10} L_{d_{10}} \frac{i_{r_{1}} \cdot i_{r_{2}}}{R_{r} \cdot \cos \beta_{4}} \dot{x} i_{d_{10}}+\psi_{f_{10}} p_{10} \frac{i_{r_{1}} \cdot i_{r_{2}}}{R_{r} \cdot \cos \beta_{4}} \dot{x}+r_{s_{10}} i_{q_{10}}=u_{q_{10}} \\
& L_{d_{6}} i_{d_{6}}-p_{6} L_{q_{6}} \frac{2 \pi i_{z} \cdot \tan \beta_{5}}{S} \dot{x} i_{q_{6}}+r_{s_{6}} i_{d_{6}}=u_{d_{6}} \\
& L_{q_{6}} i_{q_{6}}+p_{6} L_{d_{6}} \frac{2 \pi i_{z} \cdot \tan \beta_{5}}{S} \dot{x} i_{d_{6}}+\psi_{f_{6}} p_{6} \frac{2 \pi i_{z} \cdot \tan \beta_{5}}{S} \dot{x}+r_{s_{6}} i_{q_{6}}=u_{q_{6}} \\
& L_{d_{11}} i_{d_{11}}-p_{11} L_{q_{11}} \frac{i_{r_{1}} \cdot i_{r_{2}}}{R_{r} \cdot \cos \beta_{5}} \dot{x} i_{q_{11}}+r_{s_{11}} i_{d_{11}}=u_{d_{11}} \\
& L_{q_{11}} i_{q_{11}}+p_{11} L_{d_{11}} \frac{i_{r 1} \cdot i_{r 2}}{R_{r} \cdot \cos \beta_{5}} \dot{x} i_{d_{11}}+\psi_{f_{11}} p_{11} \frac{i_{r_{1}} \cdot i_{r_{2}}}{R_{r} \cdot \cos \beta_{5}} \dot{x}+r_{s_{11}} i_{q_{11}}=u_{q_{11}}
\end{aligned}
$$


表 1 变高度辊模成形机各部分转动惯量 $\left(\times 10^{-4} \mathrm{~kg} \cdot \mathrm{m}^{2}\right)$

\begin{tabular}{lc}
\hline \multicolumn{1}{c}{ 名称 } & 数值 \\
\hline$X$ 向驱动电动机转动惯量 & 156 \\
$X$ 向减速器转动惯量 & 18.3 \\
$X$ 向联轴器转动惯量 & 285.3 \\
$X$ 向驱动齿轮转动惯量 & 914.7 \\
$Z$ 向驱动电动机转动惯量 & 15.1 \\
$Z$ 向减速器转动惯量 & 7.6 \\
$Z$ 向联轴器转动惯量 & 26.1 \\
$Z$ 向运动小机架滚珠丝杜转动惯量 & 93.4 \\
轧辊驱动齿轮转动惯量 & 39.34 \\
轧辊从动齿轮转动惯量 & 703.9 \\
轧辊转动惯量 & 2054.2 \\
\hline
\end{tabular}

表 2 变高度辊模成形机各电动机参数

\begin{tabular}{lcc}
\hline \multicolumn{1}{c}{ 名称 } & $X$ 向电动机 & $Z$ 向电动机/轧辊电动机 \\
\hline 定子额定电压 $/ \mathrm{V}$ & 306 & 263 \\
交直轴电感 $/ \mathrm{H}$ & 0.016 & 0.077 \\
相电阻 $/ \Omega$ & 0.73 & 4.55 \\
额定转速 $/\left(\mathrm{r} \cdot \mathrm{min}^{-1}\right)$ & 3000 & 3000 \\
额定转矩 $/(\mathrm{N} \cdot \mathrm{m})$ & 26 & 7.3 \\
永磁体磁通 $/ \mathrm{Wb}$ & 0.1 & 0.1 \\
定子电极对数 & 4 & 4 \\
转子潻滞阻尼系数 & 0 & 0 \\
\hline
\end{tabular}

\section{表 3 变高度辊模成形机其他结构参数}

\begin{tabular}{lc}
\hline \multicolumn{1}{c}{ 名称 } & 数值 \\
\hline$X$ 向减速器减速比 & 200 \\
$Z$ 向减速器减速比 & 40 \\
轧辊齿轮副传动比 & 2 \\
$X$ 向驱动齿轮分度圆半径 $/ \mathrm{mm}$ & 80 \\
轧辊半径 $/ \mathrm{mm}$ & 130 \\
$X$ 向运动机架总质量 $/ \mathrm{kg}$ & 6247 \\
$Z$ 向运动小机架与自适应机架总质量 $/ \mathrm{kg}$ & 934 \\
$Z$ 向运动小机架滚动直线导轨副阻尼系数 & 0.04 \\
$Z$ 向运动小机架滚珠丝杜副阻尼系数 & 0.001 \\
$Z$ 向运动小机架滚珠丝杜导程 $/ \mathrm{mm}$ & 20 \\
\hline
\end{tabular}

\section{2 动力学微分方程组的载荷确定}

如图 3 所示, 零件的长度为 $876 \mathrm{~mm}$, 在长度方 向上依次分为等截面、变截面、等截面三个区域, 左右两等截面高度差 $20 \mathrm{~mm}$ 。道次间隔 $440 \mathrm{~mm}$ 。 板料厚度 $1 \mathrm{~mm}$, 折弯角度为 $84^{\circ}$, 折弯圆角半径 $5 \mathrm{~mm}$ 。

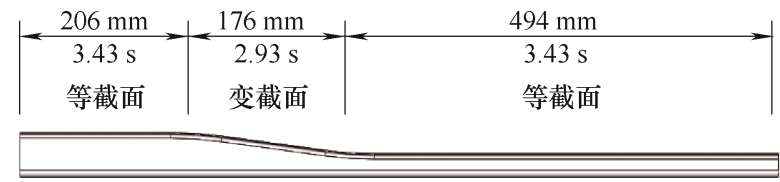

图 3 成形零件尺寸

预定 $X$ 向大机架沿 $x$ 向运动的速度为 $60 \mathrm{~mm} / \mathrm{s}$, 单个轧辊通过各段成形所需时间分别为 $3.43 \mathrm{~s}$ 、 $2.93 \mathrm{~s} 、 8.24 \mathrm{~s}$, 同一截面道次间隔 $7.33 \mathrm{~s}$ ，设第 $k$ 道 次的夹角为 $\beta_{k}$, 得到 $\beta_{k}(t)$ 的函数表达为

$$
\beta_{k}(t)= \begin{cases}0^{\circ} & (0+7.33(k-1) \leqslant t<8.24+7.33(k-1)) \\ 7^{\circ} & (8.24+7.33(k-1) \leqslant t<11.17+7.33(k-1)) \\ 0^{\circ} & (11.17+7.33(k-1) \leqslant t \leqslant 14.6+7.33(k-1))\end{cases}
$$

成形力的获取是装备研制的前提，由于定模动 辊变截面塑性变形过程非常复杂, 成形过程反作用 力矩包含弹性变形、塑性变形及摩擦阻力矩等, 无 法获得理论计算结果。利用有限元方法可以获得仿 真计算值。

选用 $1.0 \mathrm{~mm}$ 厚 QP980 高强钢板材定模动辊有 限元仿真 ${ }^{[12-13]}$, 获得如图 4 所示成形过程中五道次 轧辊所受到的反作用力矩。

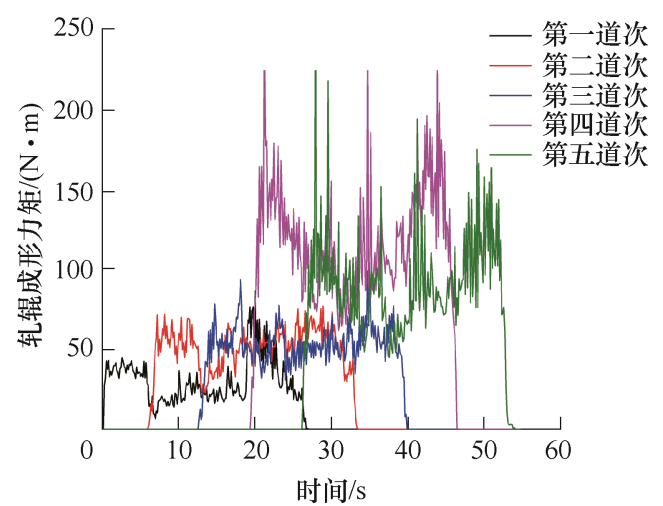

图 4 轧辊成形力矩

根据 $0 \sim 43 \mathrm{~s}$ 区间有限元计算获得离散点为 $T_{f_{k}}$ 赋值。

上述各式中的 $u_{d} 、 u_{q}$ 为电动机定子三相电压经 坐标变换后得到的交直轴上的电压, 变换矩阵的系 数及定子线电压的大小决定了 $u_{d} 、 u_{q}$ 的数值, 即

$$
\left\{\begin{array}{l}
u_{d 1}=u_{q 1}=\frac{2}{3} u_{a b c 1}=204 \sin \left(50 t+\phi_{d 1}\right)(\mathrm{V}) \\
u_{d i}=u_{q i}=\frac{2}{3} u_{a b c 2}=176 \sin \left(50 t+\phi_{d i}\right)(\mathrm{V})(i=2,3, \cdots, 11)
\end{array}\right.
$$




\section{3 方程求解与结果分析}

使用龙格库塔法, 选取合适的步长, 装备启动 时对应系统变量初始值为零。在 MATLAB 环境求 解式(27), 获得定模动辊变高度辊模成形系统各运 动参数 (加速度、速度、位移) 及电动机电流随时 间变化规律。

$X$ 向大机架各运动参数随时间的变化规律如 图 5 所示。

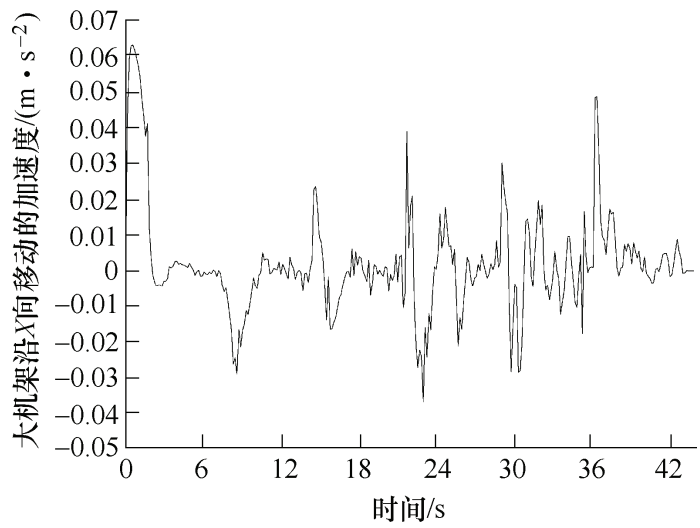

(a) 加速度

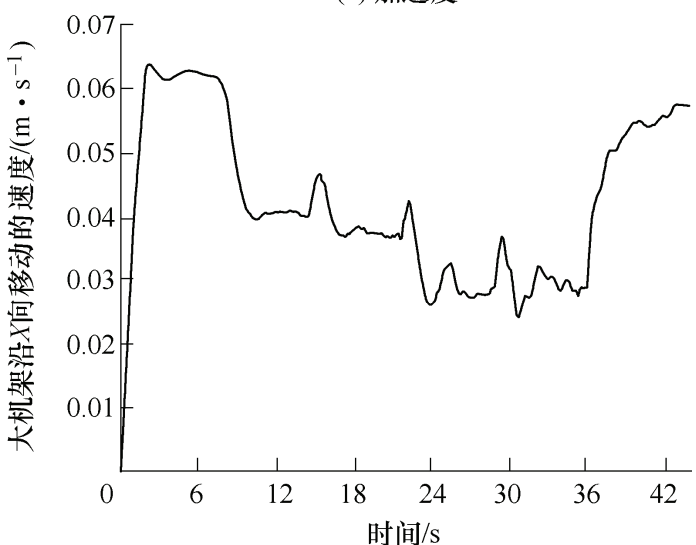

(b) 速度

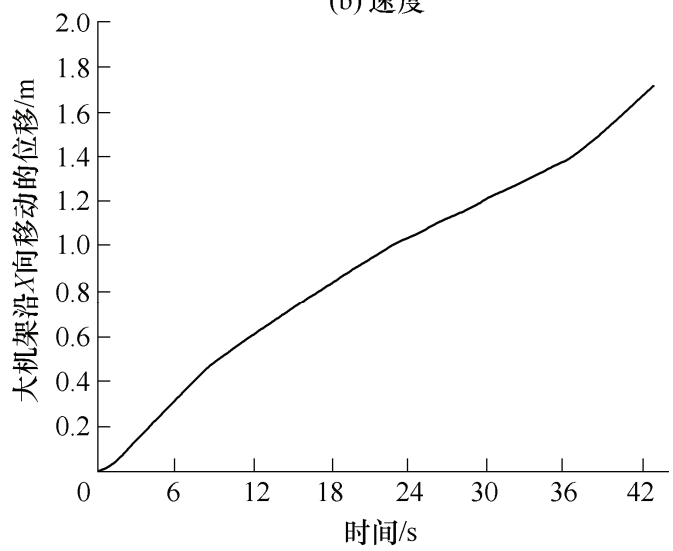

(c) 位移

图 $5 X$ 向大机架沿 $x$ 向的运动图像

$Z$ 向运动小机架沿 $z$ 向的各运动参数随时间的 变化规律如图 6 所示。

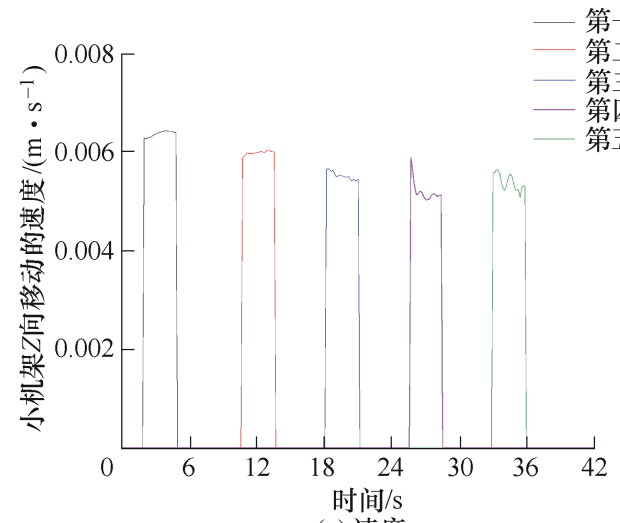

(a) 速度

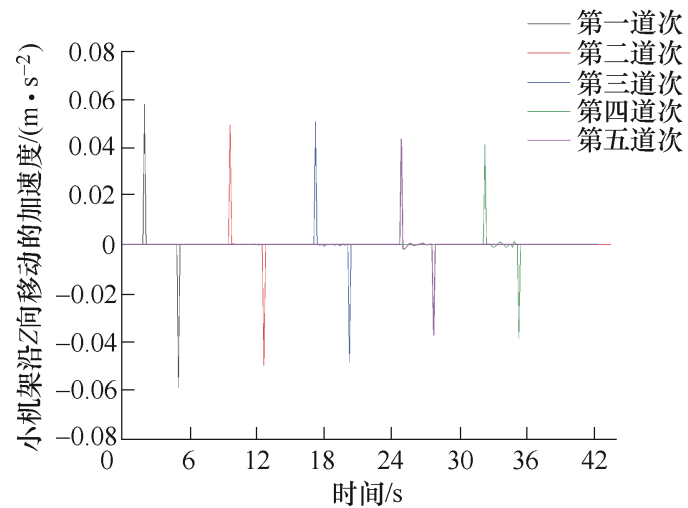

(b) 加速度

图 $6 Z$ 向运动小机架沿 $z$ 向运动图像 各伺服电动机的三相电流如图 7 所示。

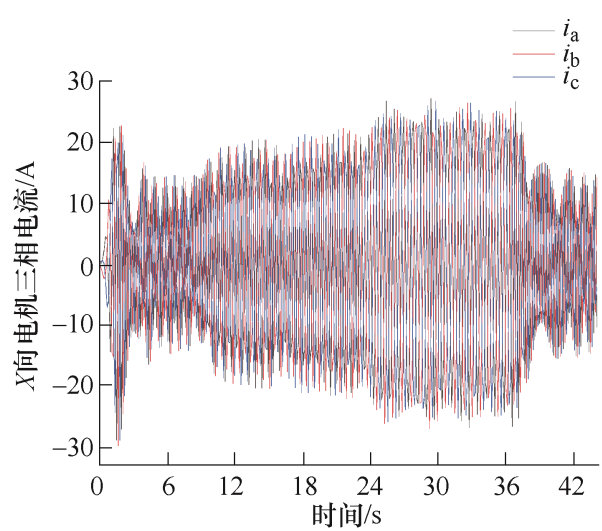

(a) $X$ 向驱动电机三相电流

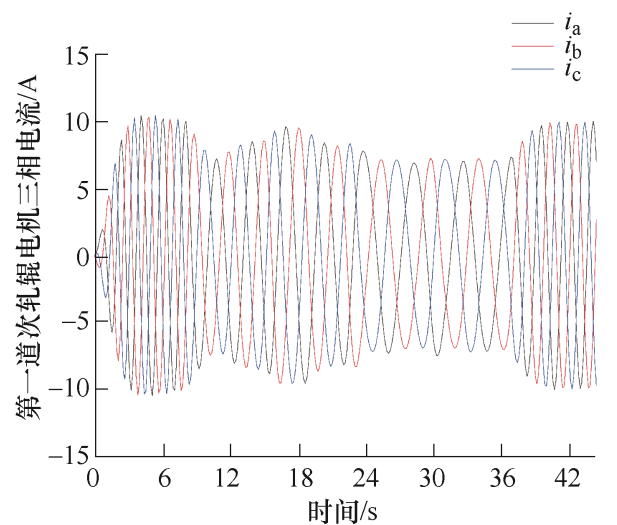

(b) 第一道次轧辊驱动电机三相电流 


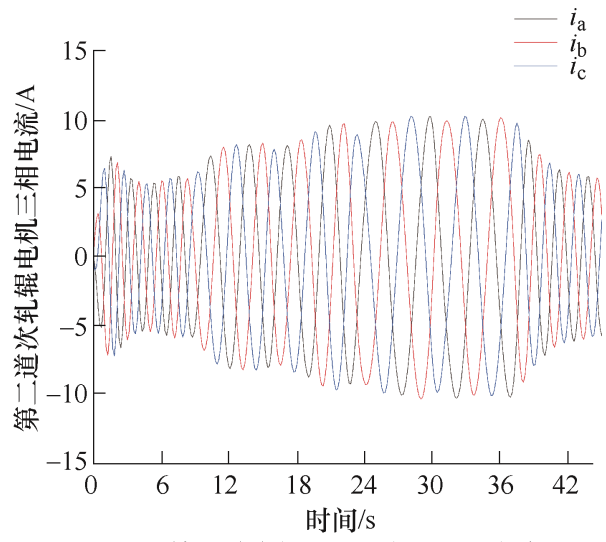

(c) 第二道次轧辊驱动电机三相电流

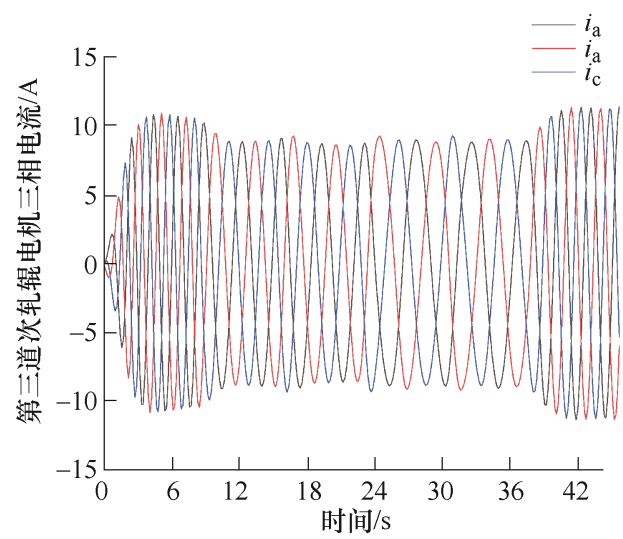

(d) 第三道次轧辊驱动电机三相电流

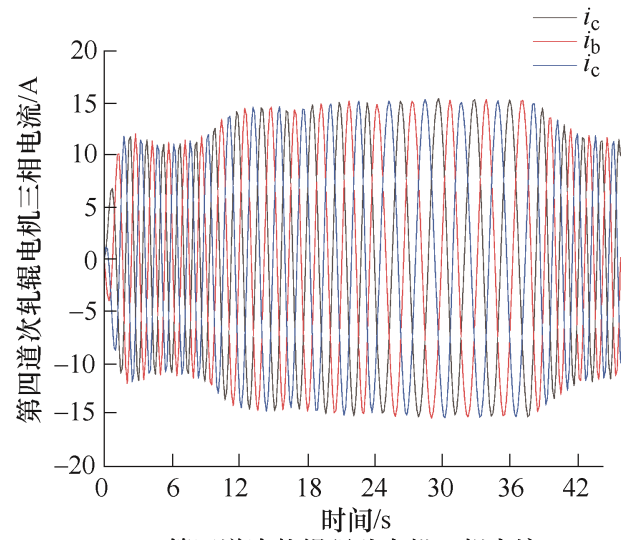

(e) 第四道次轧辊驱动电机三相电流

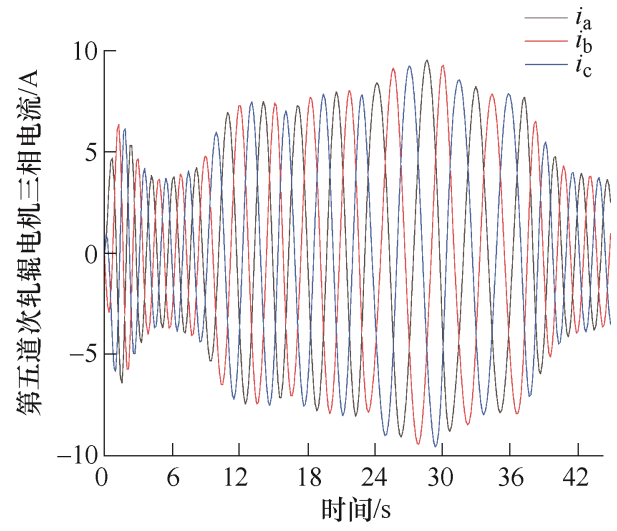

(f) 第五道次轧辊驱动电机三相电流

图 7 各驱动电动机三相电流图像
五个道次轧辊 $Z$ 向驱动电动机三相电流如图 8 所示。

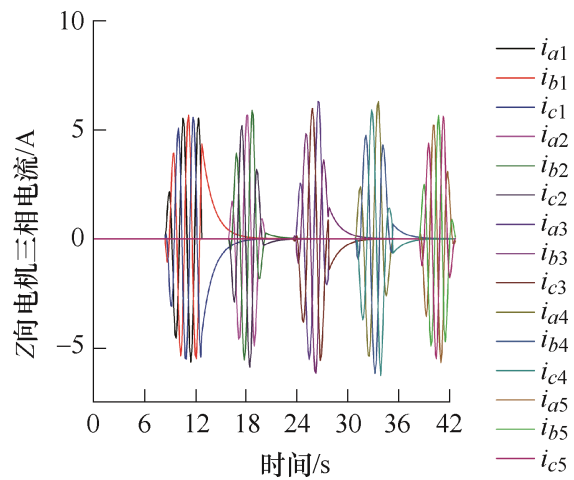

图 8 五个道次轧辊 $Z$ 向驱动电动机三相电流

由图 $5 \mathrm{a}$ 可知, 定模动辊成形过程负载明显影响 $X$ 向驱动电动机的电流, 轧辊所受反作用力矩在 0 $7.33 \mathrm{~s}$ 阶段较小, $19 \mathrm{~A}$ 左右, 25 36 s 较大, 约为 $30 \mathrm{~A}$; 由图 7c 可知, 第四道次轧辊驱动电动机的电 流在五个道次中峰值最大约为 $15 \mathrm{~A}$ 。由图 8 可知负 载对 $Z$ 向轧辊驱动电动机的电流影响较小, 其中第 四道次驱动电动机的电流最大, 约为 $5.85 \mathrm{~A}$ 。依据 各电动机所需电流, 选定系统驱动电动机:

$X$ 向驱动电动机额定功率为 $204 \times 30=6120 \mathrm{~W}$

轧辊驱动电动机功率为 $176 \times 15=2640 \mathrm{~W}$

$Z$ 向驱动电动机功率为 $176 \times 5.85=1029.6 \mathrm{~W}$

$X$ 向大机架速度是装备所有协同运动的基准, 为保障获得均匀速度, $X$ 向驱动电动机选用所需功 率 $6120 \div 0.75=8130 \mathrm{~W}$, 并以控制算法保障大机架 速度均匀。

\section{5 试验验证}

根据以上分析结果，研制出世界上第一条辊模 成形生产线，如图 9 所示。以 $1.0 \mathrm{~mm}$ 厚 QP980 板 材某轿车前纵梁变高度构件为定模动辊成形对象进 行成形试验, 构件如图 10 所示。读取部分交流伺服 电动机旋转编码器的角速度换算成对应速度值进行 试验验证。

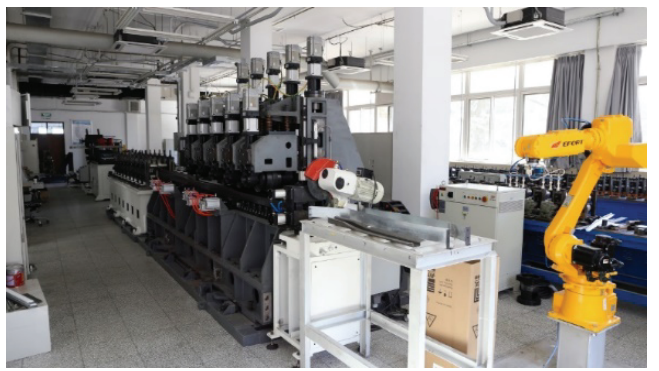

图 9 定模动辊生产线 


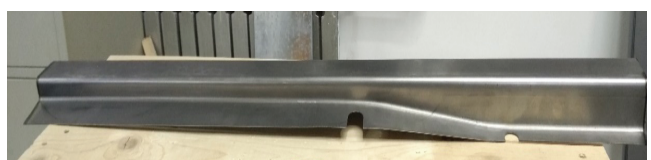

图 10 前纵梁变高度构件

实验结果与上述计算结果对比如下图 $11 \sim 15$ 所示。

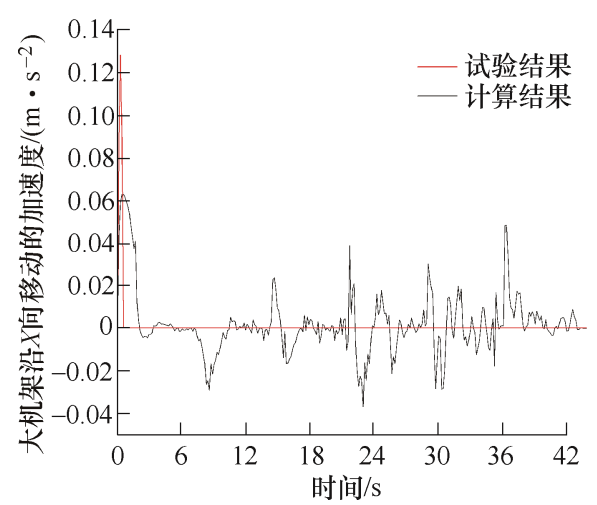

图 11 大机架 $x$ 向加速度试验对比

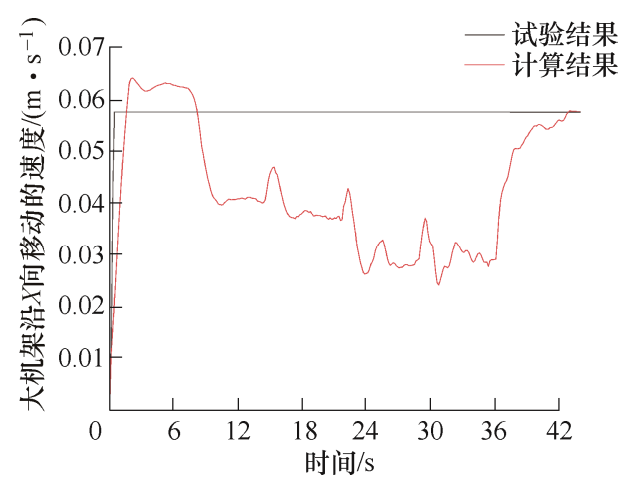

图 12 大机架 $x$ 向速度试验对比

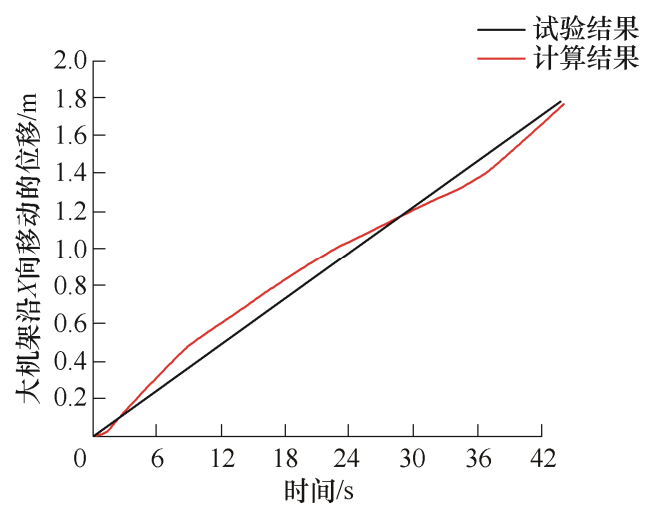

图 13 大机架 $x$ 向位移试验对比

研制的装备增大了大机架伺服电动机功率并采 用控制算法确保速度的均匀性, 因此启动时加速度 峰值大于计算值并快速减为零。当速度加速到设定 的 $60 \mathrm{~mm} / \mathrm{s}$ 后保持匀速。按照所建立的系统动力学 微分方程求解的理论计算值基本规律符合定模动辊
成形装备试验结果。理论计算结果随载荷变化呈现 相应变化, 在成形力矩增大时伺服电动机电流增大, 运动速度减小。计算结果符合系统动力学规律。

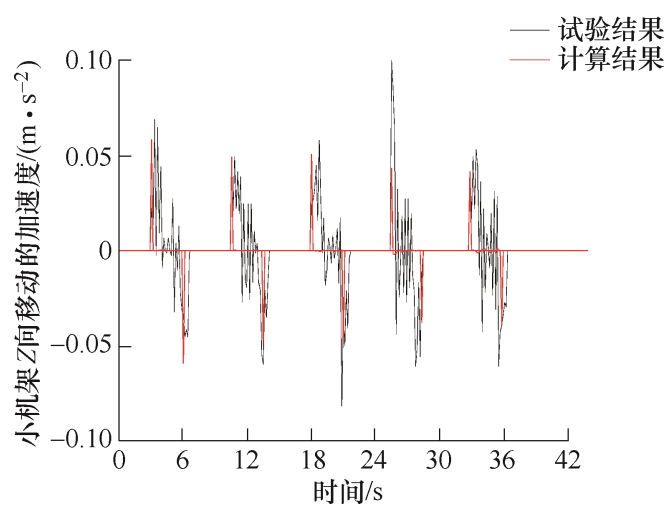

图 14 小机架 $z$ 向速度试验对比

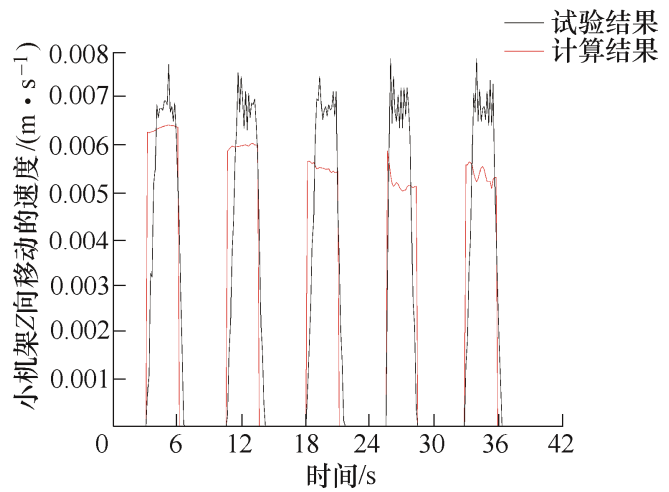

图 15 小机架 $z$ 向速度试验对比

\section{6 结论}

本文针对定模动辊变高度辊弯成形装备, 开展 驱动电动机、传动机构、模具-轧辊系统与成形高强 钢板材组成的机电系统动力学理论研究, 揭示系统 运动机理, 获得系统设计参数及性能参数, 实现定模 动辊成形装备研发。通过研究可得如下结论。

（1）根据能量原理, 建立包含装备机构、模具轧辊系统与成形高强钢板材组成的高性能精确成形 装备机电系统动力学微分方程组, 是有效的新方法。

（2）机电系统动力学分析所需要的载荷, 可以 通过有限元分析获得五个道次轧辊组成形过程的反 作用力和反作用力矩。

(3) 在 Matlab 环境采用 Runge-Kutta 法求解系 统微分方程, 获得系统成形过程全方位动态特性数 值解。

（4）依据求解结果, 以大机架运动速度为基准 确定装备所有协同运动的驱动电动机额定功率，并 以控制算法保障成形速度均匀, 为装备研发提供设 


\section{计参数值。}

(5) 利用研制的定模动辊生产线, 针对 $1.0 \mathrm{~mm}$ 厚 QP980 板材进行某型乘用车前纵梁变高度构件 制造, 试验验证基于能量原理建立的定模动辊机电 系统动力学分析方法正确。可以获得包含驱动-机构辊模-成形构件的全系统多物理场动力学分析结果, 为复杂机电系统动力学分析提供可行的方法。

\section{参 考 文 献}

[1] GROCHE P, ARND Z, BERNER S, et al. Development and verification of a one-step-model for the design of flexible roll formed parts[J]. International Journal of Material Forming, 2011, 4(1): 371-377.

[2] ONAH, SHO R, NAGAMACHI T, et al. Development of flexible cold roll forming machine controlled by PLC[R]. Toyohashi, Japan: Proceeding of the 13th International Conference on Metal Forming, 2010.

[3] PARALIKAS J, SALONITIS K, CHRYSSOLOURIS G, et al. Optimization of roll forming process parameters a semi-empirical approach[J]. Adv. Manuf. Technol., 2010, 47(1): 1041-1052.

[4] YAN Jun, LI Qiang, WU Jianxin. Electromachanical dynamics analysis and simulation on roll forming equipment based on linear motor[J]. International Journal of Modelling and Simulation, 2014, 34(3): 126-133.

[5] NA Risu, LI Qiang. Dynamic analysis of double racks gear 3D roll forming machine[J]. High Technology Letters, 2014, 20(4): 429-435.

[6] NA Risu , LI Qiang. Dynamical modeling and optimization of the roll forming machine based on the particle swarm optimization with negative gradient[J]. Sensors and Transducers, 2013, 159(11): 307-312.

[7] 陈晓宇. 多道次定模动辊机电系统动力学分析[D]. 北 京: 北方工业大学, 2020.

CHEN Xiaoyu. Electromechanical dynamics analysis of multi-pass roll-die forming machine[D]. Beijing: North
China University of Technology, 2020.

[8] LI Qiang. Flexible roll-die forming machine for variable cross-section parts: The United States of America, US9776227B2 [P]. 2017-10-03.

[9] 王振范, 刘相化. 能量理论及其在金属塑性成型中的应 用[M]. 北京: 科学出版社, 2009 .

WANG Zhenfan, LIU Xianghua. Energy principle and it's application in metal forming[M]. Beijing: Science Press, 2009.

[10] 朱越坤. 考虑成形力的定模动辊机电系统动力学分 析[D]. 北京: 北方工业大学, 2019.

ZHU Yuekun. System dynamical analysis on flexible roll-die forming machine with forming force[D]. Beijing: North China University of Technology, 2019.

[11] 温熙森, 邱静, 陶俊勇. 机电系统分析动力学及其应 用[M]. 北京: 科学出版社, 2003 .

WEN Xisen, QIU Jing, TAO Junyong. Analytic dynamics of electromechanical systems and its applications[M]. Beijing: Science Press, 2003.

[12] JIAO Jingsi, ROLFE B, MENDIGUREN J, et al. An analytical approach to predict web-warping and longitudinal strain in flexible roll formed sections of variable width[J]. International Journal of Mechanical Science, 2015, 90(1): 228-238.

[13］曾国, 来新民, 于忠奇, 等. 多道次辊弯成形动力显式 仿真的虚拟速度 $[\mathrm{J}]$. 上海交通大学学报, 2008, 42(9): 1429-1432.

ZENG Guo, LAI Xinmin, YU Zhongqi, et al. The virtual speed in dynamic explicit FEM simulation for multi-stand roll forming $[\mathrm{J}]$. Journal of Shanghai Jiao Tong University, 2008, 42(9): 1429-1432.

作者简介: 阳振峰, 男, 1987 年出生, 硕士, 讲师。主要研究方向为机 电装备研制、机电一体化技术。

E-mail: yzf@ncut.edu.cn

李强(通信作者), 男, 1963 年出生, 博士, 教授, 博士研究生导师。主 要研究方向为机电系统动力学分析与优化。

E-mail: liqiang@ncut.edu.cn 